\title{
COMPARISON OF TWO EXPERIMENTS ON RADIATIVE NEUTRON DECAY
}

\author{
Khafizov R.U..$^{\text {a }}$, Tolokonnikov S.V. ${ }^{\text {a }}$, Solovei V.A. ${ }^{\text {, }}$, Kolhidashvili M.R. ${ }^{\text {b }}$ \\ ${ }^{a}$ RRC Kurchatov Institute, 123182 Moscow, Russia \\ ${ }^{\mathrm{b}}$ Petersburg Nuclear Physics Institute, 188350 Gatchina, Russia
}

\begin{abstract}
Over 10 years ago we proposed an experiment on measuring the characteristics of radiative neutron decay in papers $[1,2]$. At the same time we had published the theoretical spectrum of radiative gamma quanta, calculated within the framework of the electroweak interactions, on the basis of which we proposed the methodology for the future experiment $[3,4]$. However, because we were denied beam time on the intensive cold neutron beam at ILL (Grenoble, France) for a number of years, we could only conduct the experiment in 2005 on the newly opened FRMII reactor of Technical University of Muenchen. The main result of this experiment was the discovery of radiative neutron decay and the measurement of its relative intensity B.R. $=(3.2 \pm 1.6) \cdot 10^{-3}$ with C.L. $=99.7 \%$ for radiative gamma quanta with energy over $35 \mathrm{kev}[5,6]$. Over a year after our first announcement about the results of the conducted experiment, "Nature" [7] published a letter asserting that its authors have also measured the branching ratio of radiative neutron decay B.R. $=(3.13 \pm 0.34) \cdot 10^{-3}$ with C.L. $=68 \%$ and gamma quanta energy from 15 to $340 \mathrm{kev}$. This article aims to compare these two experiments. It is shown that the use of strong magnetic fields in the NIST (Washington, USA) experiment methodology not only prevents any exact measurement of the branching ratio and identification of radiative neutron decay events, but also makes registration of ordinary neutron decay events impossible.
\end{abstract}

PACS numbers: 13.30.Ce; 13.40.Hq; 14.20.Dh

Among the many branches of elementary decay with charged particles in the final state, the radiative branch, where the decay occurs with the creation of an additional particle - the gamma quantum, is usually the most intensive, as the relative intensity ( or branching ratio B.R. ) of this mode is determined by the fine structure constant $\alpha$ of $10^{-2}$ order of magnitude. This decay branch is well established and was investigated for almost all elementary particles. However, the radiative decay of the free neutron

$$
n \rightarrow p+e+\bar{v}+\gamma
$$

was not discovered, and all the experiments were aimed at the study of the ordinary neutron decay branch. We conducted the first experiment on the discovery of this rare neutron decay branch in 2002 on the intensive cold neutron beams at ILL (Grenoble, France), where we received a limit for the relative intensity of this rare decay branch: B.R. $<6.9 * 10^{-3}$ ( $90 \%$ C.L.) [8]. This value exceeds the theoretical value we calculated and published only in by a few times. This fact, in turn, means that in our experiment of 2002 we came very close to discovering the radiative mode of neutron decay. For reasons outside of our control we did not receive beam time on intensive cold neutron beam at ILL for a number of years and were able to continue our experiment only in 2005, after we received beam time at the newly opened FRMII 
reactor in Munchen. In this experiment we identified the events of radiative neutron decay and measure its relative intensity B.R. $=(3.2 \pm 1.6) \cdot 10^{-3}$ with C.L. $=99.7 \%$ and gamma quanta energy over $35 \mathrm{kev}[5,6]$. A year after our discovery of the radiative neutron decay, a NIST experimental group published the results of their experiment on the study of the radiative neutron decay [7] in Nature, with their own value of B.R. $=(3.13 \pm 0.34) \cdot 10^{-3}$ with C.L. $=68 \%$ and gamma quanta energy from 15 to $340 \mathrm{kev}$. This work is dedicated to the comparison of these two experiments.

Our group carried out calculations of the neutron radiative spectrum in the framework of standard electroweak theory were carried out about ten years ago [1-4]. The calculated branching ratio for this decay mode as a function of the gamma energy threshold is shown in Figs. 1 and 2. The branching ratio for the energy region investigated here, i.e. over $35 \mathrm{keV}$, was calculated to be about $2 \cdot 10^{-3}$ ( gamma energy threshold $\omega$ on Fig. 1 and 2 is equal to $35 \mathrm{keV}$ [3] ). Given this rather large branching ratio of about two per thousand, it is in principle not a difficult task to measure it. In practice, however, a rather significant background, mainly caused by external bremsstrahlung emitted by the decay electrons when stopped in the electron detector, has to be overcome. In this experiment this was achieved with the help of a triple coincidence requirement between the electron, the gamma-quantum and the recoil proton. The presence of such a coincidence is used to identify a radiative neutron decay event, whereas an ordinary neutron beta decay is defined by the coincidence of an electron with a recoil proton. The latter coincidence scheme is routinely used to measure the emission asymmetry of electrons in the decay of polarized neutrons [9-10]. Besides the non-correlated background one also has to deal with a correlated background of bremstrahlung gamma-quanta that fully simulates the desired fundamental radiative decay process searched for. This correlated background is caused by bremsstrahlung emission of the electron traveling through the electron detector and is quite significant even when the thickness of this detector is limited to only a few $\mathrm{mm}$. It cannot be eliminated by requiring a triple coincidence of the electron, the photon and the proton. However, calculations [3] show that the radiative emission of a photon in neutron decay is not in the forward direction with respect to the electron emission direction, as in the case of bremsstrahlung, but reaches a maximum intensity at an angle of $35^{\circ}$ (Fig. 3). It was this property of radiative neutron decay that led us to use the space solution and register gamma quanta and electrons by different detectors, located at an angle to each other. To achieve this, we constructed a segmented electron-gamma detector with a $35^{\circ}$ angle between the sections for electron and gamma detection to reduce this background.

The experimental set-up is shown schematically in fig. 4. The intense cold neutron beam passes through a rather long neutron guide in which is installed a collimation system made of LiF diaphragms, placed at regular distances of 1 meter. The neutrons enter the vacuum chamber (1) through the last diaphragm (9) that is located directly before the decay zone. This zone is observed by three types of detector: the micro channel plate (MCP) proton detector (3), the electron detector (13) consisting of a 7 $\mathrm{cm}$ diameter and $3 \mathrm{~mm}$ thick plastic scintillator, and six gamma detectors (11) that are located on a ring centered around the electron detector and which consist of photomultiplier tubes each covered with a layer of $\mathrm{CsI}(\mathrm{Tl})$ scintillator. The thickness of these $7 \mathrm{~cm}$ diameter $\mathrm{CsI}(\mathrm{Tl})$ scintillators is $4 \mathrm{~mm}$ and has been selected so as to have a $100 \%$ detection efficiency for photons. The six gamma detectors (11) surround the electron detector (13) (cf. the lower part of fig. 4) at an angle of $35^{\circ}$ and are shielded from it by $6 \mathrm{~mm}$ of lead (12). By requiring a coincidence between the electron detector and any of the gamma detectors the bremsstrahlung background can 
in principle be overcome completely, because bremsstrahlung emission occurs only in the section that registers the electron. In this case, part of the statistics is lost, of course, as can be seen from figure 3. However, the neutron beam intensity of $10^{12} \mathrm{n} / \mathrm{s}$ in our experimental chamber is sufficiently enough to compensate for that loss and still allows for a good count rate. Recoil protons, formed in the decay zone, pass through a cylindrical time of flight electrode (7) in the direction of the proton detector (3) and are focused onto this detector with the help of spherical focusing electrodes (2). The focusing electrostatic field between the high voltage spherical and cylindrical electrodes (2) and (7) is created by the grids (5) and (6) at one side and by the proton detector grid (4), at ground potential, at the other side. It is important to note that the recoil protons take off isotropic from the decay point. In order not to lose the protons emitted towards the electron detector, an additional grid (10) is added on the other side of the decay volume.

The start signal that opens the time windows for all detectors is the signal from the electron, registered in the electron detector (13). For an event to be considered as a radiative neutron decay event there have to be simultaneous signals from the electron detector (13) and one of the gamma detectors (11), followed by a delayed signal from the proton detector (3). It is important to note that in the case of radiative decay, the gamma quantum in our equipment is registered by gamma detectors (11) surrounding the electron detector (13) before the electron is registered by the electron detector. In other words, electron is delayed in comparison to the radiative gamma quantum. In the future namely this fact will allow us to distinguish the peak of radiative gamma quanta in the triple coincidences spectrum. Besides these triple coincidences also double electron-proton coincidences, signaling an ordinary neutron decay event, are monitored.

It is important to note here that thanks to the LiF ceramics diaphragm system which was installed in the neutron beam line, the gamma background from the intense cold neutron beam was significantly suppressed. The background level in the gamma detector amounted to about $2.5 \mathrm{kHz}$ only (at a neutron beam intensity of $10^{10} \mathrm{n} / \mathrm{s}$ ). If the number of the diaphragms in the neutron guide were doubled, the background of the gamma detectors could be further reduced by another order of magnitude, thus becoming comparable to the noise of the photomultiplier tubes. Another important note is that in our last experiment at ILL we succeeded at obtaining a gamma background that was smaller by an order. This could be explained by the fact that on the "Mephisto" beam at FRMII we used a collimation system that was reduced in comparison to the original. Besides, our experiment was the first to be conducted on the intensive cold beam neutron "Mephisto" and as it turned out the axis of this beam went a little higher than the axis of our collimation system, which also contributed to a significant increase in the gamma background. The count rate in the electron detector was just about $100 \mathrm{~Hz}$. It is very likely that most of this count rate is due to electrons from neutron decay since the count rate in this detector almost immediately dropped to zero when the neutron beam was switched off. The value of proton detector background amounted to about 4-6 kHz, which turned out to be very sensitive to the vacuum conditions in the experimental chamber. This background is explained primarily by the presence of a high number of ions in the decay zone, which are captured by the external electric field and are registered by the proton detector (3) along with the recoil proton. ( see Fig. 4 ).

All components of the experimental set-up performed well. Electron-proton coincidences could clearly be observed, while the sectioned electron-gamma detector performed as expected. The results obtained with a vacuum less than $10^{-6} \mathrm{mbar}$, are 
presented in figures 5 and 6. We would like to emphasize that Fig. 5 and 6 of our article present experimental spectra in their raw form, as they were before any preliminary data processing or background subtraction. Fig. 5 demonstrates the summary statistics on double e-p coincidences (coincidences of electron with delayed proton), and Fig. 6 demonstrates the summary statistics on triple e-p- $\gamma$ coincidences (coincidences of electron, gamma-quantum, and delayed proton). Fig. 5 clearly shows two major peaks: one peak with a maximum in channels 99-100, which is the peak of zero or prompt coincidences $[5,6,11]$. The position of this peak marks the zero time count, namely the time when the electron detector registered the electron. The next peak visible on Fig. 5 has a maximum in channel 120, and is the peak of e-p coincidences of electron with delayed proton. An analogous situation was observed in experiments on the measurement of the correlation coefficients by two independent groups at ILL [10] and at NIST [11], and it was also mentioned at [12]. Fig. 5 shows that the total number of events in e-p coincidences peak in our experiment equals $\mathrm{N}_{\mathrm{D}}=3.75 \cdot 10^{5}$. This value exceeds the value we obtained in our previous experiment conducted on beam PF1 at ILL by two orders. It was precisely because of the low statistics volume that we could not then identify the events of radiative neutron decay, and defined only the upper B.R. limit [8]. It is very important to note that the peak of double coincidences between electron and the delayed proton is observed against a non-homogenous background: besides the homogenous ionic background, which has a value comparable to the value of the e-p coincidences peak, there is an obvious peak in channels 99-100. In essence, this peak is a response peak to the time spectrum of electron registration, which contains just one peak in channels 99-100, signifying the time when the electron detector registered the electron. In the future we will see that the peak of triple coincidences appears against a non-homogenous background with not one, but two response peaks.

The remaining peaks on Fig. 5 are small, with just seven peaks distinct from the statistical fluctuations. These occurred because of the noises in electric circuit of the FRMII neutron guide hall. There are no other physics-related reasons for their occurrence. These peaks appeared and disappeared depending on the time of day, reaching their maxima during the work day and disappearing over the weekends. Such behavior was observed throughout the experiment as we collected statistics. Since the nature of these seven small peaks is in no way related to radiative and ordinary decay, we did not emphasize them in our article

Fig. 6 of triple coincidences clearly shows three peaks, and the leftmost peak with the maximum in channel 103 is connected to the peak of the radiative gamma-quanta in question, as this gamma-quantum is registered by the gamma detectors in our equipment before the electron. Comparing Fig. 5 and 6, it becomes clear that if we ignore the first leftmost peak with the maximum in channel 103 in Fig. 6 , the spectrum of double e-p coincidences will resemble the spectrum of triple e-p- $\gamma$ coincidences. The peak with the maximum in channel 106 on Fig. 6 is connected to the left peak of false coincidences on Fig. 5, and the peak with the maximum in channel 120 on Fig. 6 is connected to the right peak of e-p coincidences on Fig. 5. The emerging picture becomes obvious when one uses a standard procedure, introducing a response function for gamma channel $\mathrm{R}_{\gamma}\left(\mathrm{t}, \mathrm{t}^{\prime}\right)$, which is necessary also for calculating the number of triple radiative coincidences $\mathrm{N}_{\mathrm{T}}$ in radiative peak. Using this method of response function, one can confidently define our double-humped background: the narrow peak with the maximum in channel 106 on Fig. 6 is the response to the narrow peak of zero coincidences in channels 99-100 on Fig. 5, and 
the second peak in this double-humped background on Fig. 6 is the response to the peak in channels $117-127$ on Fig. 5.

Of course, the width of these response peaks is greater than the width of the two original peaks on Fig. 5, the width of the narrow peak increases more, the distance between the peaks themselves diminishes, and the narrow peak moves to the right towards the wider peak more than this wide peak moves in opposite direction to the left . Such behavior is described using the standard method of response function, and there is nothing unusual about it. Indeed, for real detectors and electronics the response function is always not local, which explains all these deformations. In our case this non-local behavior is connected to the fact that the rise time of the gamma signal from gamma detectors is on average 150-200 ns (which equals to 6-8 time channels on Fig. 5 and 6), and the rise time of the electron signal from the electron detector equals to $10 \mathrm{~ns}$. This difference explains, in particular, the shift of the peaks on Fig. 6: the peak with maximum in channel 106, which is the response to the peak of zero coincidences, is shifted to the right (the side of delay) in comparison to the peak of zero coincidences in channel 99-100 on Fig. 5.

Here it is also appropriate to remark on another peculiarity observed throughout the experiment and which emphasizes the physics-related nature of the peak in channel 103 on Fig. 6. As noted above, the noise peaks on Fig. 5 were not stable, and neither were the small peaks in channels 96 and 116 on Fig. 6. Besides, if the small noise peaks in the spectrum of double coincidences disappeared, the small peaks in channels 96 and 116 in the spectrum of triple coincidences disappeared as well. The radiative peak in channel 103 was stable throughout, it never "migrated" to a different channel and its growth was stable, regularly collecting the same number of events during the same stretch of time!

As for the wide, almost indistinguishable peak in channel 165 on Fig. 6, its influence on radiative peak in channel 103 is negligible. Its nature is in no way related to the researched phenomenon, so we do not discuss it in our article.

After analyzing the spectra with the help of the non-local response function we finalize the average value for the number of radiative neutron decays $\mathrm{N}_{T}=360$ with a statistics fluctuation of 60 events. B.R. can be expressed through ratio $N_{T}$ to $N_{D}$ as $B R$ $=\mathrm{k}\left(\mathrm{N}_{\mathrm{T}} / \mathrm{N}_{\mathrm{D}}\right)$, where coefficient $\mathrm{k}=3.3$ is geometrical factor what we can calculate by using not isotropic emission of radiative gamma-quanta Fig. 3. With the number of observed double e-p coincidences $N_{D}=3.75 \cdot 10^{5}$, triple e-p- $\gamma$ coincidences $\mathrm{N}_{\mathrm{T}}=360$, one then deduces the value for radiative decay branching ratio of $(3.2 \pm 1.6) \cdot 10^{-3}$ (99.7 \% C.L.) with the threshold gamma energy $\omega=35 \mathrm{keV}$ The average B.R. value we obtained deviates from the standard model, but because of the presence of a significant error $(50 \%)$ we cannot make any definite conclusions. Precision level must be increased. According to our estimates, we will be able to make more definite conclusions about deviation from the standard electroweak theory at the precision level of less than $10 \%$.

Unfortunately, the other report [7] published a year after our article and dedicated to experimental study of the radiative neutron decay mode with which we are comparing our results publishes neither initial spectra with raw experimental data on double coincidences of $\beta$-electron with recoil proton, nor data on triple coincidences of the electron-proton pair with the radiative gamma-quantum. In and of itself, this undermines confidence in this report. The only initial experimental data published in the report are the shape of the pulses from the combined electron-proton detector and the gamma-quanta detector (see Fig. 7 from [7] ). The difference between the NIST experiment and our experiment becomes immediately apparent. First and foremost, it 
is the time scale: in our spectra, the scale is measured in nanoseconds, while in the other experiment the scale is in microseconds. Besides, we used three types of detectors, each of which registered its own particle: one detector for the electrons, one for the protons, and six identical detectors for the radiative gamma-quanta (see Fig. 4). The duration of the front pulse from the electron and proton detectors is 10 nanoseconds in our experiment and 100 times greater than that in the NIST experiment, in the order of $1 \mu \mathrm{s}$. The rise time of gamma signal from our gammadetectors is on average $150 \mathrm{~ns}$, and from avalanche diode on the NIST equipment greater than $10 \mathrm{~ms}$, besides that the diode pulse arrives with significant noise, which makes the thickness of the front pulse line equal to more than $0.5 \mu$ s (see the red line on Fig. 7 from [7]).

All of this leads to our factual time resolution being two orders better than the resolution achieved in the NIST experiment. However, as the two experiments used equipment which was practically the same in size and smaller than 1 meter, the choice of the time scale is a matter of principle. Given this geometry, it is impossible to get microsecond signal delays from all of the registered charged particles, i.e. electrons and protons. In this light, it is surprising that the peak identified by the authors of the NIST report [7] as the peak of radiative gamma-quanta, is shifted by 1.25 microseconds to the left. The expectation that magnetic fields of several tesla in magnitude delay all electrons and protons, are absolutely ungrounded.

Indeed, the electron and proton velocities depend only on the kinetic energy of these particles. Fig. 8 shows the dependence of the ratio of electron velocity $\mathrm{v}$ to the speed of light $\mathrm{c}$ on the ratio of electron kinetic energy $\mathrm{T}_{\mathrm{e}}$ to the electron mass energy $\mathrm{m}_{\mathrm{e}} \mathrm{c}^{2}$. It is evident that the speed of $\beta$-electron, the kinetic energy of which is measured in hundreds of $\mathrm{keV}$, is comparable with the speed of light and in one microsecond should travel hundreds of meters, and not one meter. The length of trajectory in magnetic fields also does not depend on the magnitude of the magnetic field, and rather depends only on the direction of electron emission in relation to the direction of the magnetic field. Fig. 9 shows the dependence of the ratio of trajectory length 1 to the distance from the point of decay to detector $L$ on angle $\theta$ between the direction of electron emission and the direction of the magnetic field. This dependence is rather smooth and also does not depend on the magnitude of the magnetic field. As demonstrated by this graph, the ratio is $1 / \mathrm{L}<2$ for electrons emitted at angle $\theta$ less than $60^{\circ}$ and $1 / \mathrm{L}<10$ for $\theta<85^{\circ}$. As $\beta$-electron emission is isotropic, it means that practically all electrons travel from the point of decay to the detector in tens of nanoseconds, but not in microseconds.

When we discuss the delay of beta-electrons in relation to ratiative gamma-quanta, we would like to emphasize the following point in methodology. We wrote about this directly in our article published in JETP Letters [5], when discussing the reasons for the non-locality of the response function. This aspect concerns the method of determining the location of the radiative peak in relation to the time of electron registration. The beginning of the pulse from the radiative gamma-quantum obviously predates the beginning of the pulse from the electron detector. But, as we just explained, this difference is extremely small and is measured in nanoseconds, and not microseconds. However, noises do not allow to exactly determine the location of the beginning for the gamma-quantum signal, so we determined the location of the radiative peak using the true-constant-fraction ( TCF ) method in our coincidences scheme, using a constant fraction discriminator ( CFD ) [13]. As the fronts of the gamma detector signals are flatter than the fronts of the electron detector signals, the time-pickoff signal from the gamma quanta appeared later in relation to the signal of 
the electron time-pickoff signal, so the location of the radiative peak should be to the right of the electron registration time. In other words, the radiative peak in our coincidences scheme should be delayed in relation to the electron registration time by the electron detector. In our case (see Fig. 6), the radiative peak is located to the right of the 100th channel by a value less than the rise time of the gamma-detector signal and is positioned with precision of $25 \mathrm{~ns}$. The response to the peak of momentary coincidences in the spectrum of triple coincidences ( the peak with the maximum in channel 106 on Fig. 6 ) is also delayed and is shifted to the right of the 100th channel. At the same time, in the spectrum of triple coincidences on Fig. 6 the radiative peak is located, as it should be since the radiative quantum arrives at the gamma detector before all other particles, on the left slope of the response peak, and not on the right. Thus, we obtained the location of our radiative peak exactly where we expected it. As it has already been noted above, the movement of the response peaks and their widths are accounted for in the analysis of experimental data by the introduction of the nonlocal response function.

Now let us consider the other coincidences pair, namely the coincidences of electrons and recoil protons. Fig. 10 presents $t$ the dependence of the ratio of proton velocity $\mathrm{v}$ to the speed of light $\mathrm{c}$ on the ratio of proton kinetic energy $\mathrm{T}_{\mathrm{p}}$ to the proton mass energy $m_{p} c^{2}$ As the original kinetic energy of the recoil protons in the betadecay is extremely low, it is determined primarily by the potential of the electronproton detector (in our case, this is the potential of focusing electrodes). However, even in the case where this potential is equal to just $35 \mathrm{kev}$, proton velocity is still significant and is slightly less than $1 \%$ of the light velocity c. In our case, when proton kinetic energy was $25 \mathrm{keV}$, the final velocity was $0.006 \mathrm{c}$, and its average velocity along the trajectory was 0.003 c. On Fig. 6, which shows the spectrum of the electron-proton coincidences, the peak of these coincidences is located in the 120th channel, which corresponds to proton delay of $500 \mathrm{~ns}$ on average or the distance between the point of decay and the proton detector in $40-50 \mathrm{~cm}$. The estimate obtained is quite good and coincides with the real distance between the proton detector and the axis of neutron beam in our equipment with precision of ten-twenty per cent.

The presence of a strong magnetic field cannot radically change the obtained value of the recoil proton delay and change it from hundreds of nanoseconds to ten microseconds.

As the initial energy of the recoil proton is very small in comparison to electric field potential, angle $\theta$ (see Fig. 3) is now defined as the angle between the direction of the electric field and the magnetic field. As follows from the geometry of the NIST experiment equipment, the electric field is directed along the magnetic field and angle $\theta$ should be no more than a few degrees. On the other hand, as follows from our spectrum of double e-p coincidences Fig. 5, the peak of electron-proton coincidences is observed at a rather significant ionic background, the value of which is equal to the value of the electron-proton field itself. In other words, the process of ion formation is comparable in its magnitude to the process of the recoil proton formation in the neutron beta-decay. These ions may be captured by the strong magnetic field in our opponents' equipment and produce impulses delayed by ten microseconds. From this it follows that the signal from recoil protons on Fig. 11 is at the base of the electron signal the duration of which is over 1 microsecond, and so simply cannot be registered by the combined electron-proton detector in the NIST experiment equipment. The signal shown on Fig. 11 as a proton signal delayed by 10 microseconds, and is the signal from an ion captured by the magnetic and electric 
fields. From this it follows that the use of the strong magnetic fields prevents reliable identification of ordinary neutron decay events, much less radiative events, in the NIST experiment. In light of the above, the lavish praise [14] heaped on the NIST experiment cited in the article is unfounded. Moreover, the fact that the review of the work was published in the same issue as the article presenting the work [7, 14] is rather surprising.

Finally, it is necessary to note that when using strong magnetic fields it is impossible to determine the average angle between electron moment direction and the direction of radiative gamma-quantum momenta. As shown in the calculated spectrum on Fig. 3, the intensity of radiative gamma-quanta emission strongly depends on this angle, which makes comparison between theory and experimental data, obtained in magnetic fields, very problematic. In our equipment, on the other hand, the average angle between the electron and the gamma-quanta momenta is fixed in the region of maximum radiative gamma quanta emission intensity and so determines the geometric factor.

In conclusion, we would like to emphasize two main points concerning the experiment we conducted.

First, the results from the first experiment aiming to observe the as yet undiscovered radiative decay mode of the free neutron are reported. Although the experiment could not be performed under ideal conditions, the data collected still allowed one to deduce the B.R. $=(3.2 \pm 1.6) \cdot 10^{-3}(99.7 \%$ C.L. $)$ for the branching ratio of radiative neutron decay in the gamma energy region greater than $35 \mathrm{keV}$. This value is in agreement with the theoretical prediction based on the standard model of weak interactions.

Secondly, the average B.R. value we obtained deviates from the standard model, but because of the presence of a significant error $(50 \%)$ we cannot make any definite conclusions. Taking into account the fact that the experimental conditions can still be significantly optimized, an e-p coincidence count rate of 5-10 events per second is within reach. Together with the standard model prediction for the branching ratio of this decay mode, this would correspond to a triple e-p- $\gamma$ coincidence rate of several events per 100 seconds. This can easily be observed with the current experimental set-up, which is now being optimized with a view to performing such an experiment. The aim of that experiment will then not only be to establish the existence of radiative neutron beta decay, but also to study B.R. in more detail. This, in turn, would allow to discover the deviation from standard electroweak theory. According to our estimates, we will be able to make more definite conclusions about deviation from the standard electroweak theory at the precision level of less than $10 \%$.

Despite the recent disagreements [14], which we consider to be subjective in nature [15], we acknowledge the contribution of our Western colleagues Profs. N. Severijns, O. Zimmer and Drs. H.-F. Wirth, D. Rich to our experiment conducted in 2005. The authors would like to thank Profs. D. Dubbers, J. Deutsch, A.P. Serebrov, V.V. Fedorov and Drs. T. Soldner, G. Petzoldt, I. Konorov and S. Mironov for valuable remarks and discussions. We are also grateful to the administration of the FRMII, especially Profs. K. Schreckenbach and W. Petry for organizing our work. We would especially like to thank RRC President Academician E.P. Velikhov and Prof. V.P. Martem'yanov for their support, without which we would not have been able to conduct this experiment. Financial support for this work was obtained from INTAS (Project N 1-A -00115; Open 2000), the RFBR (Project N 07-02-00517). We would especially like to note the early financial support from the INTAS fund. 
Without this support neither the 2002 ILL experiment, nor the 2005 experiment at TUM which we discuss in this paper would have been possible.

\section{References}

[1] Radiative neutron beta-decay and its possible experimental realization Gaponov Yu.V., Khafizov R.U. Phys. Lett. B 379 (1996) 7-12

[2] Radiative neutron beta-decay and experimental neutron anomaly problem. By Yu.V. Gaponov, R.U. Khafizov . Weak and electromagnetic interactions in nuclei (WEIN '95): proceedings. Edited by H. Ejiri, T. Kishimoto, T. Sato. River Edge, NJ, World Scientific, 1995. 745p.

[3] About the possibility of conducting an experiment on radiative neutron beta decay R.U. Khafizov, N. Severijns Proceedings of VIII International Seminar on Interaction of Neutrons with Nuclei (ISINN-8) Dubna, May 17-20, 2000 (E3-2000-192), 185-195 [4] Angular distribution of radiative gamma quanta in radiative beta decay of neutron. Khafizov R.U. Physics of Particles and Nuclei, Letters 108 (2001) 45-53

[5] R.U. Khafizov, N. Severijns, O. Zimmer et al. JETP Letters 83 (2006) p. 5

[6] Discovery of the neutron radiative decay R.U. Khafizov, N. Severijns et al. Proceedings of XIV International Seminar on Interaction of Neutrons with Nuclei (ISINN-14) Dubna, May 24-25, 2006

[7] J. Nico, et al. Observation of the radiative decay mode of the free neutron. Nature, v. 444 (2006) p. 1059-1062

[8] M. Beck, J. Byrne, R.U. Khafizov, et al., JETP Letters 76, 2002, p. 332

[9] B. G. Yerozolimsky, Yu. A. Mostovoi, V P. Fedunin, et al., Yad. Fiz. 28, 98 (1978) [Sov. J. Nucl. Phys. 28, 48 (1978)].

[10] I.A. Kuznetsov, A. P. Serebrov, I. V. Stepanenko, et al., Phys. Rev. Lett., 75 (1995) 794.

[11] L.J. Lising, S.R. Hwang, J.M. Adams, et al., Phys. Rev. C. v.6, 2000, p. 055501

[12] J.Byrne, R.U. Khafizov, Yu.A. Mostovoi, et al., J. Res.Natl. Inst. Stand. Technol.

110, p.415, (2005).

[13] T.J. Paulus, IEEE Transactions on Nuclear Science, v.NS-32, no.3, June, p.1242, 1985.

[14] N.Severijns, Nature, v. 444 (2006) p. 1014-1015.

[15] N. Severijns, et al., e-print arXiv:nucl-ex/0607023.

[16] R.U. Khafizov, V.A. Solovei, e-print arXiv:nucl-ex/0608038. 


\section{$\underline{\text { Figure captions }}$}

Fig. 1. The expected standard model branching ratio for radiative neutron beta decay (summed over all gamma energies larger than the threshold gamma energy $\omega$ ) as a function of $\omega$ (from [1-4]).

Fig. 2. The expected standard model branching ratio for radiative neutron beta decay (summed over all gamma energies larger than the threshold gamma energy $\omega$ ) as a function of $\omega$, for the low energy part of the spectrum (from [1-4]).

Fig. 3. Dependence of the radiative decay spectrum on the angle $\Xi$ between the photon and the electron momenta (upper curve for a threshold gamma energy of 25 $\mathrm{keV}$, lower curve for a threshold gamma energy of $50 \mathrm{keV}$ ) (from $[3,4])$.

Fig. 4. Schematic lay-out of the expemrimental set-up.

(1) detector vacuum chamber, (2) spherical electrodes to focus the recoil protons on the (at $18-20 \mathrm{kV}),(3)$ proton detector, (4) grid for proton detector (at ground potential), (5) \& (6) grids for time of flight electrode, (7) time of flight electrode (at $18-20 \mathrm{kV})$, (8) plastic collimator $(5 \mathrm{~mm}$ thick, diameter $70 \mathrm{~mm}$ ) for beta-electrons, (9) LiF diafragms, (10) grid to turn the recoil proton backward (at 22-26 kV), (11) six photomultiplier tubes for the $\mathrm{CsI}(\mathrm{Tl})$ gamma detectors, (12) lead cup, (13) photomultiplier tube for the plastic scintillator electron detector.

Fig. 5. Timing spectrum for e-p coincidences. Each channel corresponds to $25 \mathrm{~ns}$. The peak at channel 99-100 corresponds to the prompt ( or zero ) coincidences. The coincidences between the decay electrons and delayed recoil protons (e-p coincidences) are contained in the large peak centered at channel 120.

Fig. 6. Timing spectrum for triple e-p-g coincidences. Each channel corresponds to 25 ns. In this spectrum, three main peaks in channels 103, 106 and 120 can be distinguished. The leftmost peak in 103 channel among these three main peaks is connected with the peak of radiative decay events.

Fig. 7. The red line shows the shape of pulses from the gamma detector, built on avalanche diodes, and the blue line shows the shape of pulses from the combined electron-proton detector ( see ref. [7] )

Fig. 8. Relationship between the ratio of electron speed $v$ to the speed of light $\mathrm{c}$ and the ratio of kinetic electron energy Te to the energy of its mass $m_{e} c^{2}$.

Fig. 9. Relationship between the ratio of the trajectory length 1 of charged particles moving in the magnetic field to distance L between the point of decay and the detector and the angle between the velocity of this particle and the direction of the magnetic field $\theta$.

Fig. 10. Relationship between the ratio of proton velocity $\mathrm{v}$ to speed of light $\mathrm{c}$ and the ratio of kinetic proton energy $T_{p}$ to its mass $m_{p} c^{2}$.

Fig. 11. The signal from the decay proton has to be delayed by less than one microsecond, which is why it is located at the base of the electron pulse and so cannot be registered by the combined electron-proton detector. The pulses that are delayed by longer than 1 microsecond are pulses not from decay protons, as it was indicated in ref. [7], but rather from ions, formed in the decay zone. 
B.R.

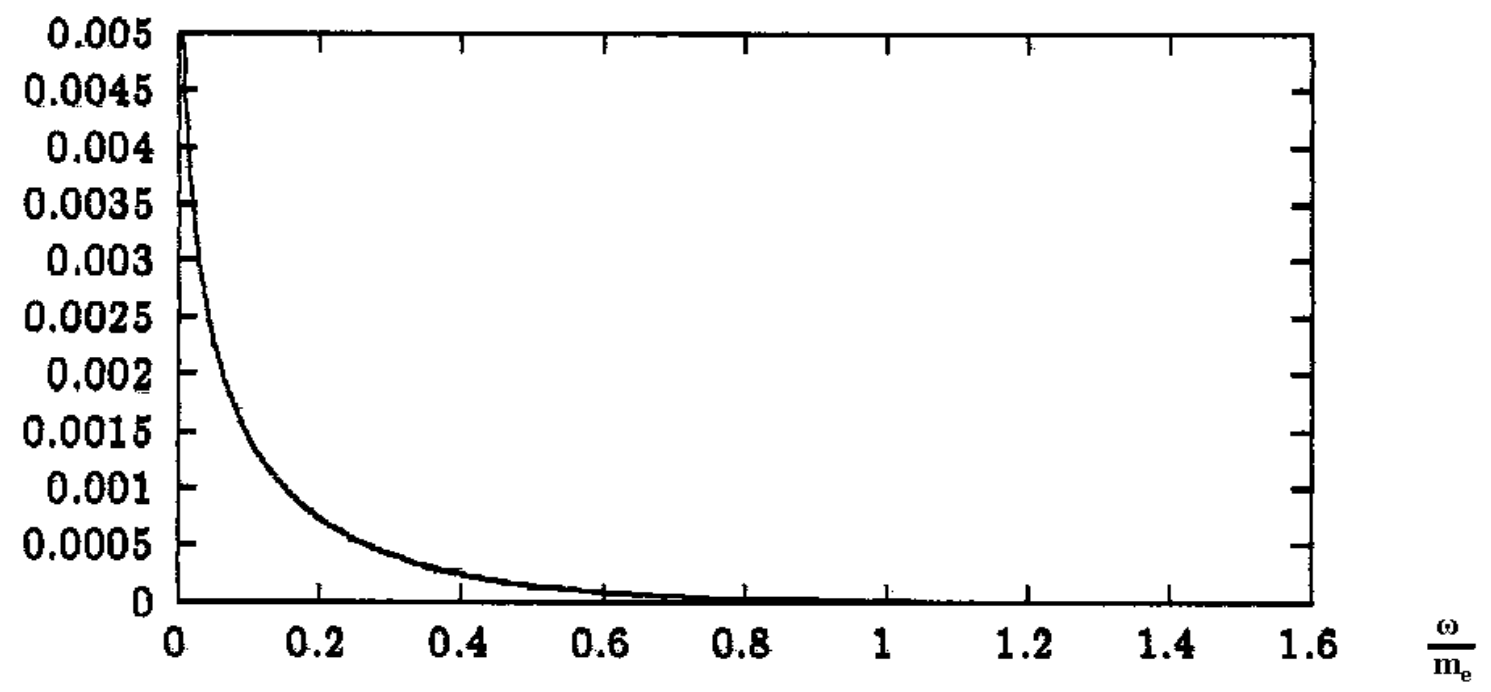

Fig. 1 


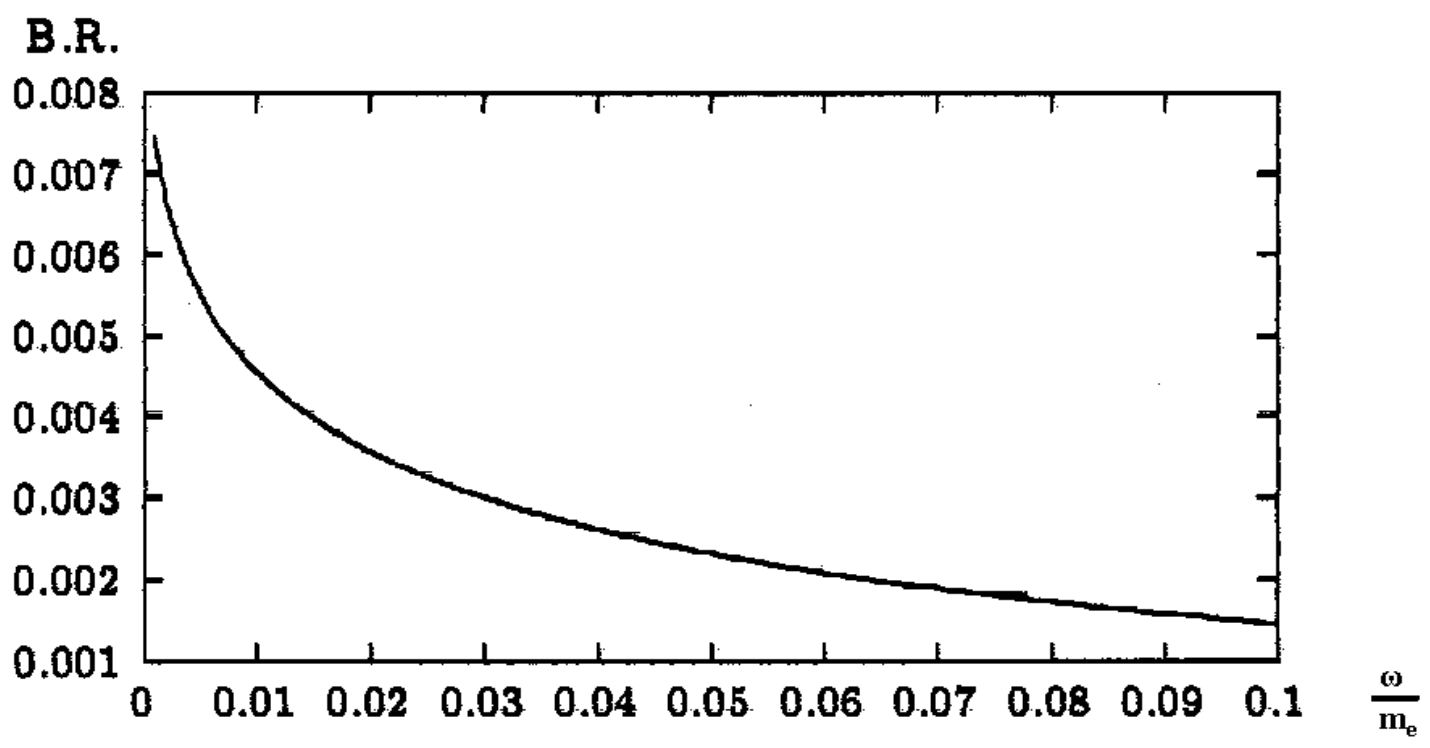

Fig. 2 


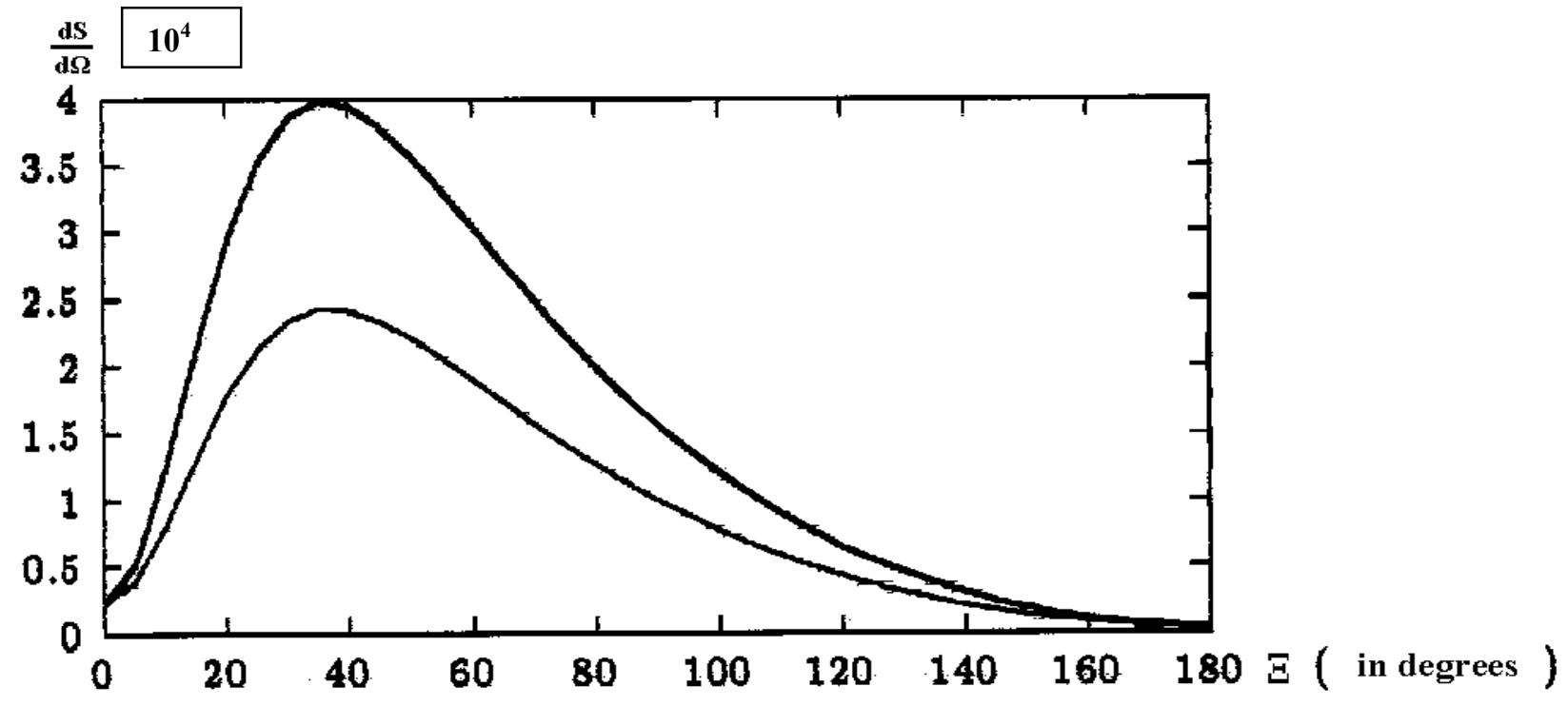

Fig. 3 


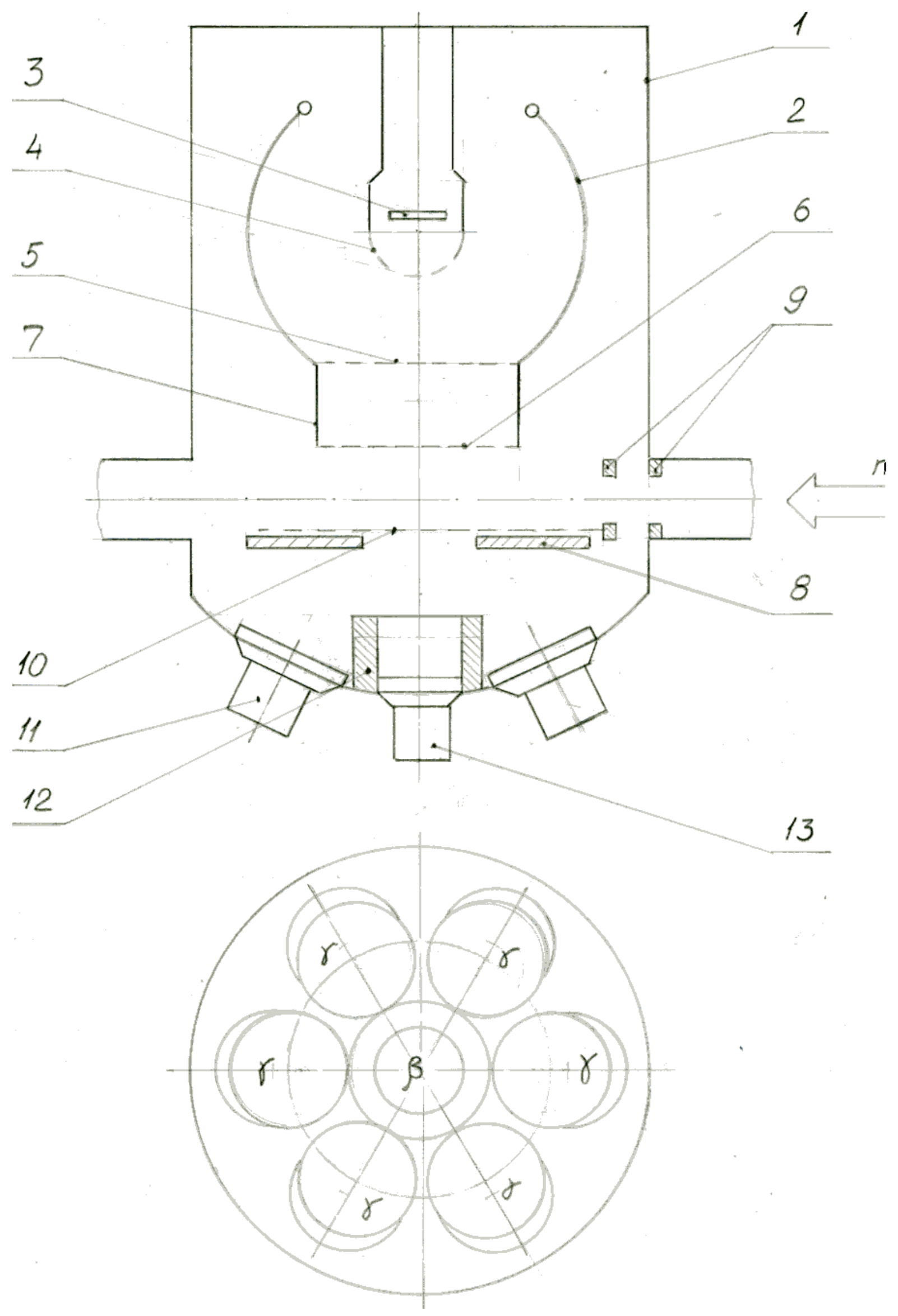

Fig. 4 
Counts

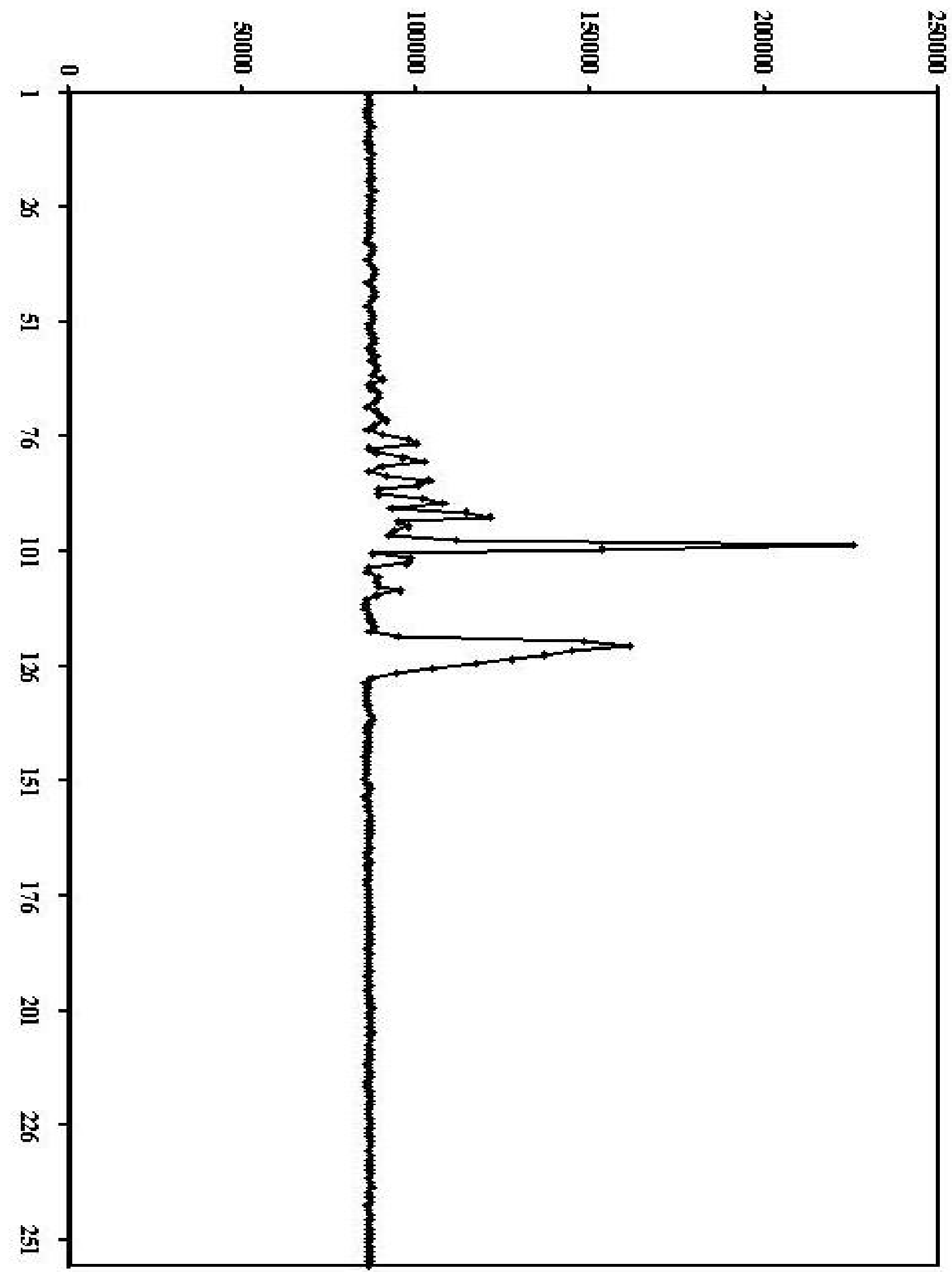

Fig. 5 


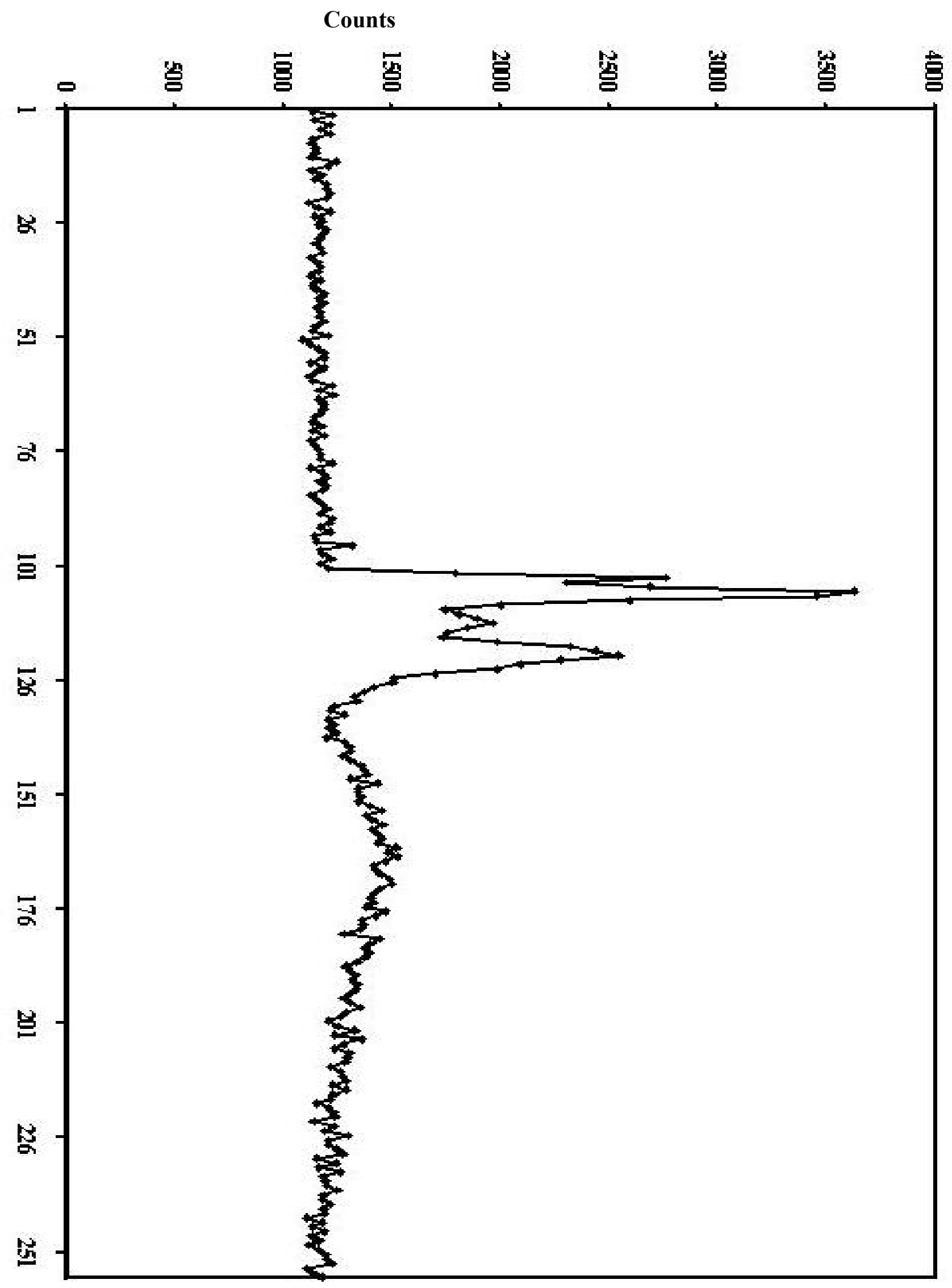

Fig. 6 


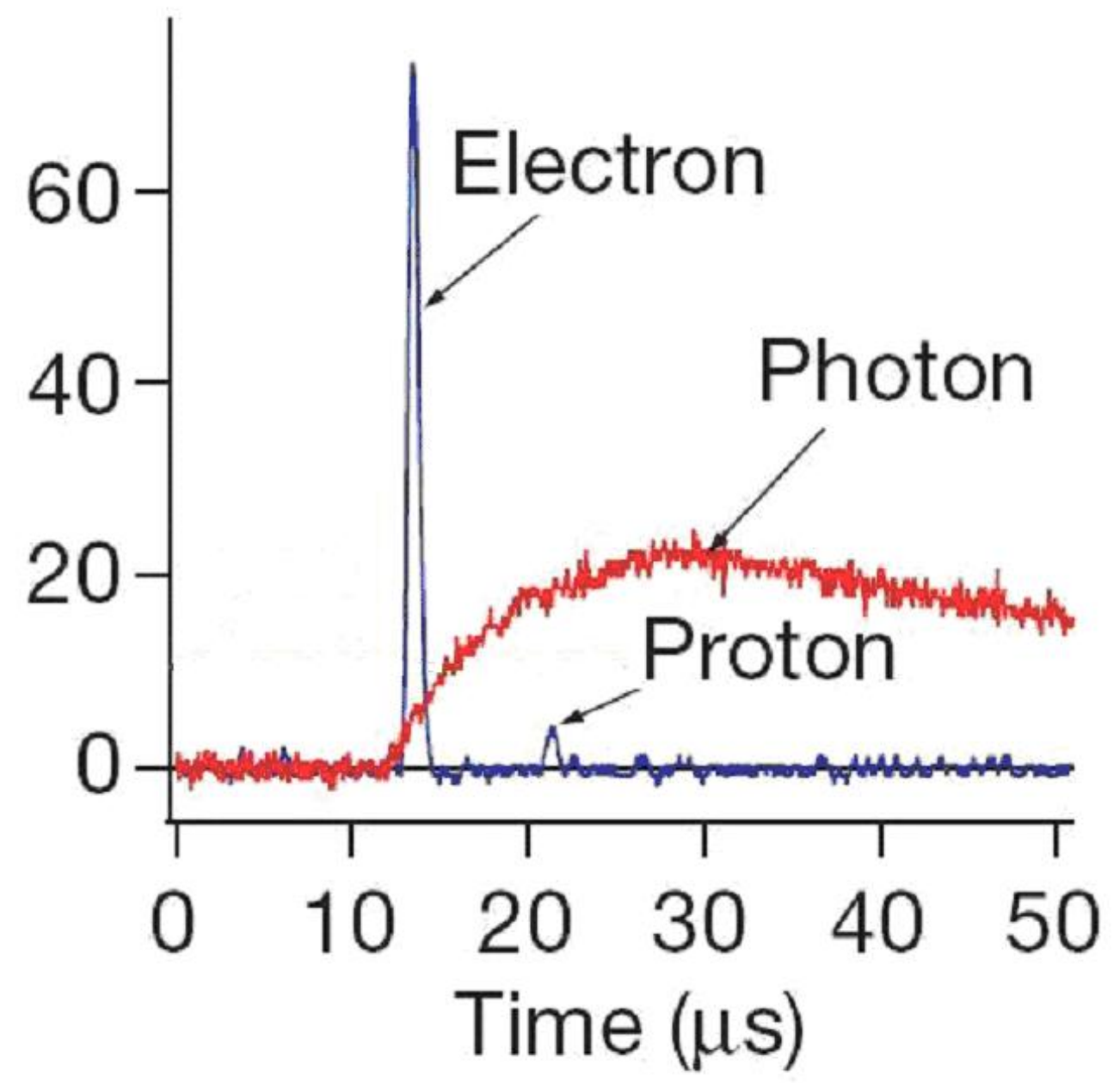

Fig. 7 


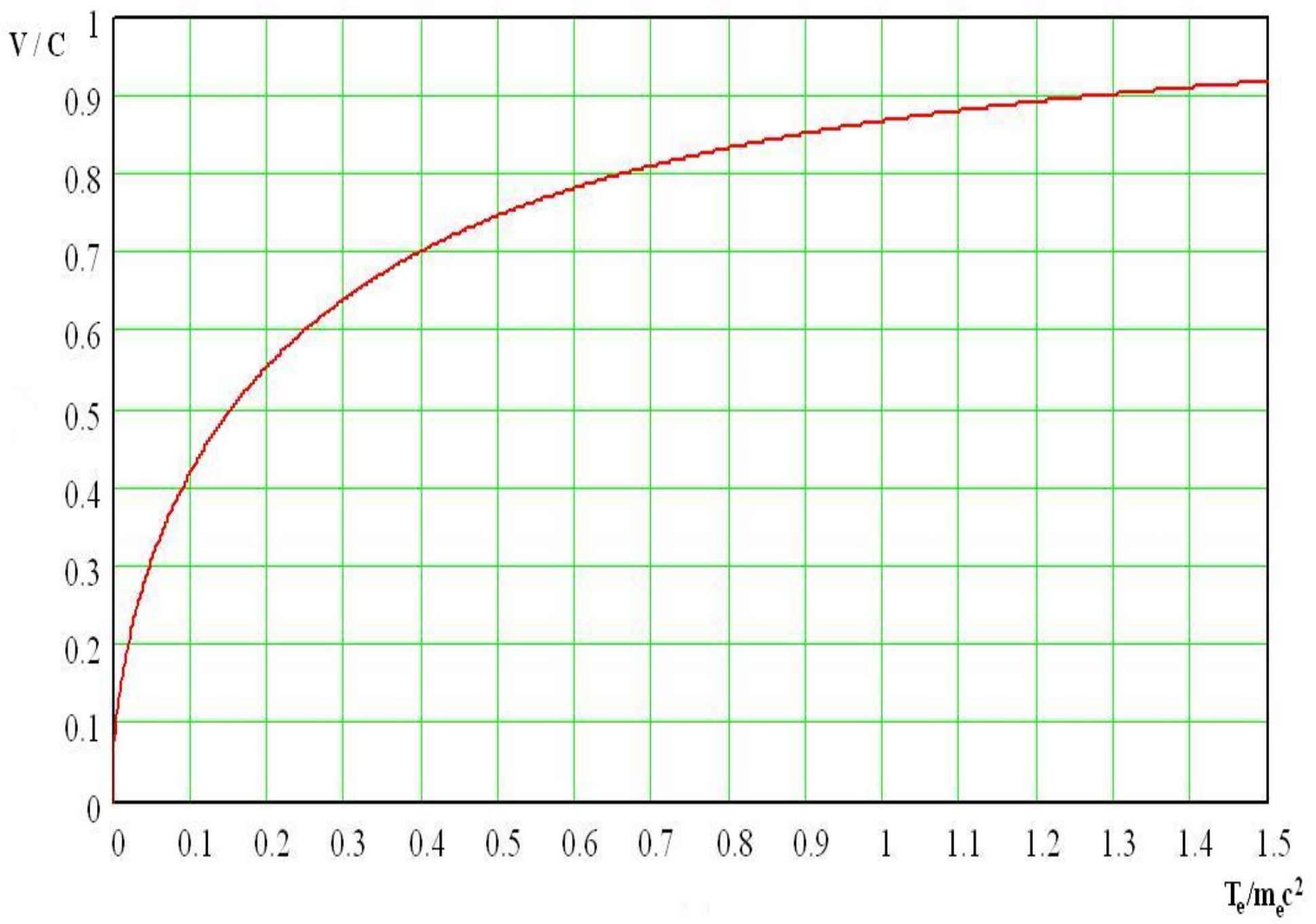

Fig. 8 


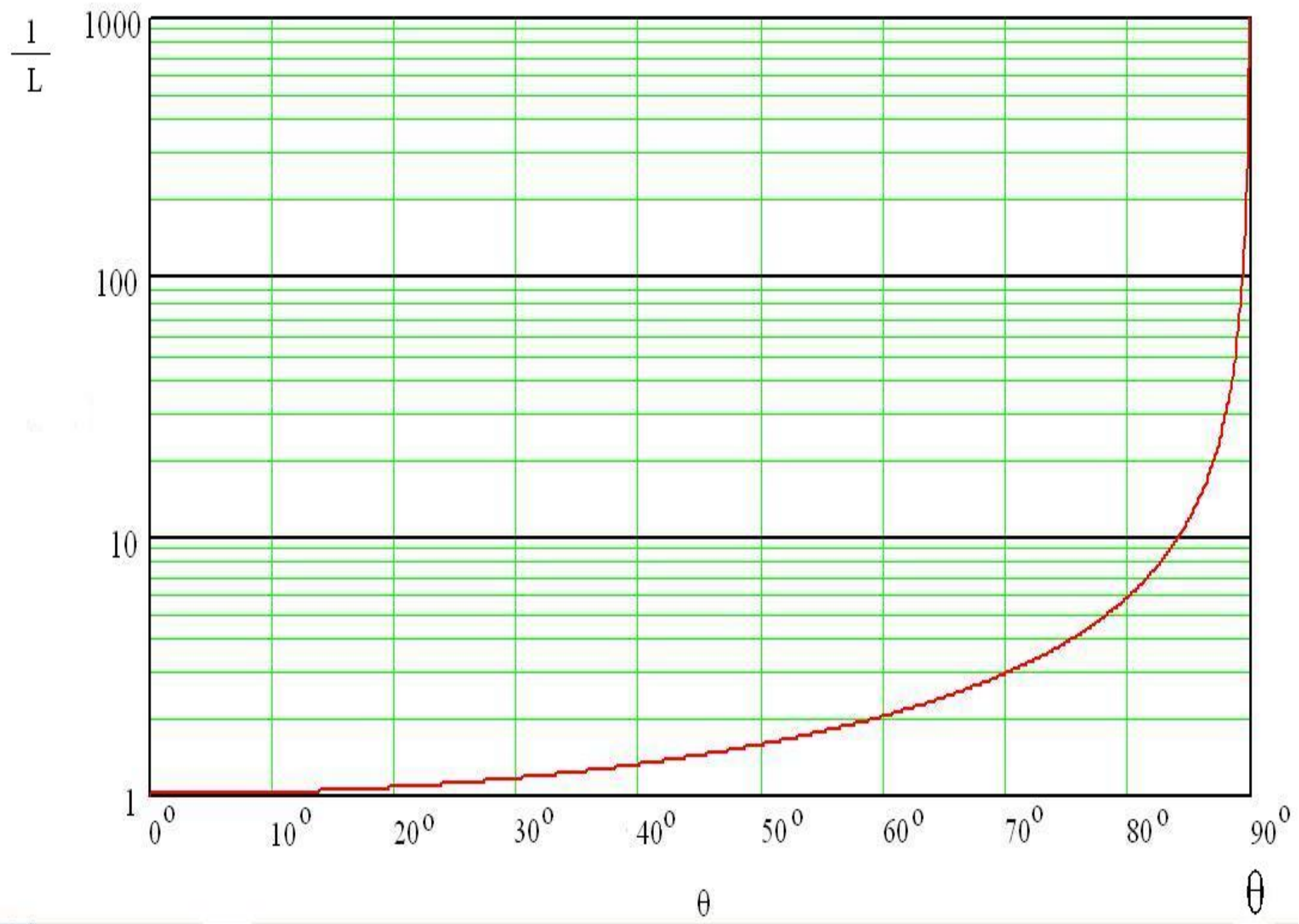

Fig. 9 
$\mathrm{V} / \mathrm{C} 0.02$

0.018

0.016

0.014

0.012

0.01

0.008

0.006

0.004

0.002

0

$0 \quad 1 \cdot 10^{-5} 2 \cdot 10^{-5} 3 \cdot 10^{-5} 4 \cdot 10^{-5} 5 \cdot 10^{-5} 6 \cdot 10^{-5} 7 \cdot 10^{-5} 8 \cdot 10^{-5} 9 \cdot 10^{-5} 1 \cdot 10^{-}$ $\mathrm{T}_{\mathrm{p}} / \mathrm{m}_{\mathrm{p}} \mathrm{c}^{2}$

Fig. 10 


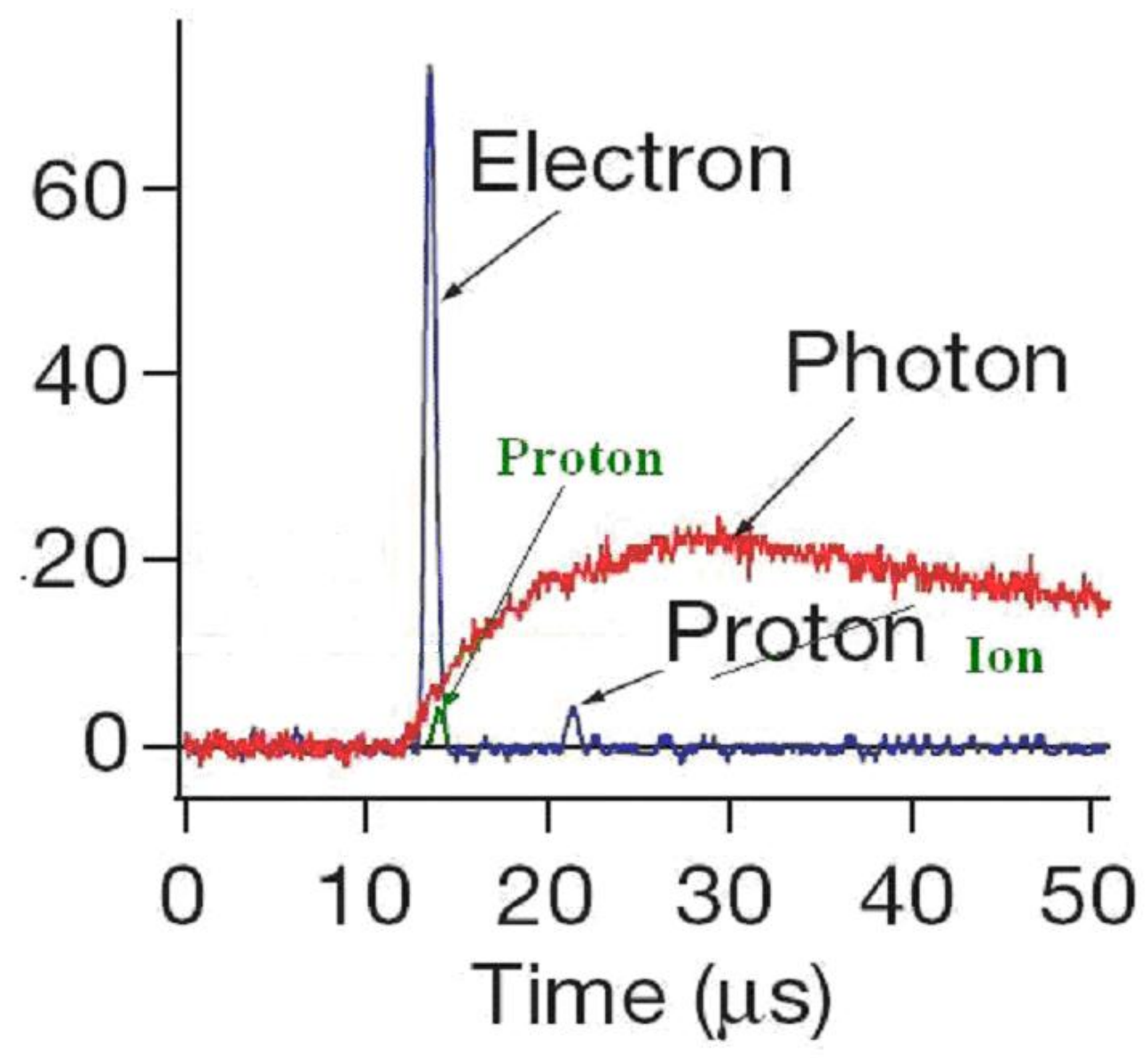

Fig. 11 